\title{
A short communication nutritional observation study
}

Asmaa Fathi Hamouda ${ }^{1,2,3^{*}}$, Eman Kamal Omar Mahamed ${ }^{4}$, Walaa Omar Abdel Aziz Zeina ${ }^{5}$, Sara Ahmed Khazaei ${ }^{2}$ Amal Abootalep Basheery ${ }^{2}$, Haneen Mohammed Mubarki², Sahar Jaduh Ogdi ${ }^{2}$, Manal Ahmed Sholan, Rafeef Mohammed Edrees , Asma Housin Khazaei, Hajer Fawaz Khardli, Amani Mohammed Al-Oqebi ${ }^{2}$, Afrah Abdullah Saad Aldosari ${ }^{2}$, Mariam Mohamed Majrashi ${ }^{2}$, Wejdan Ali Mohammed ${ }^{2}$, Khawla Mosa Mashi²

${ }^{1}$ Department of biochemistry, Faculty of Science, University of Alexandria, Alexandria, Egypt.

${ }^{2}$ MLT Department, Faculty of Applied Health Sciences, Jazan University, Jazan, Kingdom of Saudi Arabia.

${ }^{3}$ Poison Control and Medical Forensic Chemistry Center, Ministry of Health, Jazan, Kingdom of Saudi Arabia.

${ }^{4}$ Clinical pathology specialist, Al-Haram Hospital Giza Egypt.

${ }^{5}$ Faculty of science Tanta University, Egypt.

*Corresponding Author: Asmaa Fathi Hamouda, Ph.D., (2013) Alexandria University, Egypt, Assistant Professor in the Umm Al-Qura University, Saudi Arabia (2014-2016), and in Assistant Professor Faculty of Applied Health Sciences, Jazan University University, Jazan University, Saudi Arabia.

E-mail:asmaakingdom1@yahoo.com, AFMOUSTAFA@jazanu.edu.sa

Received date: October 30, 2020; Accepted date: November 07, 2020; Published date: November 11, 2020

Citation: Asmaa F. Hamouda, Eman K. O.Mahamed, Walaa O. A. A. Zeina, Sara A. Khazaei, Amal A. Basheery, Haneen M. Mubarki, Sahar J. Ogdi, Manal A. Sholan, Rafeef M. Edrees, Asma H. Khazaei, Hajer F. Khardli, Amani M. Al-Oqebi, Afrah A. Saad Aldosari, Mariam M. Majrashi, Wejdan A. Mohammed, Khawla M. Mashi (2020) A short communication nutritional observation study, J. Pharmaceutics and Pharmacology Research 3(2); DOI: 10.31579/2693-7247/020

Copyright: ( ) 2020, Asmaa Fathi Hamouda, This is an open access article distributed under the Creative Commons Attribution License, which permits unrestricted use, distribution, and reproduction in any medium, provided the original work is properly cited.

\section{Abstract}

Obesity is the most prevalent nutritional dysfunction in society and the vital risk determinant for several relevant disorders. The first step of obesity assessment is calculated anthropometric measures and, secondly, measure biological and nutritional profiles. The current purpose is to investigate the relationship between BMI with biochemical profile, Vitamin $\mathrm{B} 12, \mathrm{Fe}$, and cortisol in the volunteer's population.

There is an appositively connection between body mass index (BMI) with cortisol, liver and kidney profile, fasting blood sugar (FBS), Cholesterol, triglyceride, and low-density lipoprotein (LDL) and negative relationship between BMI and Vitamin B12, Fe, and high-density lipoprotein (HDL) in the present study. There is a significant elevation of cortisol, lipid profile, FBS, liver, and kidney function with BMI in the current work in females more than males compared to a reference range, and a significant decrease in Fe and vitamin B that can be due to diet habits, BMI, malabsorption in obesity condition, hormones affect or genetic variations between male and female. That will need further investigation.

Keywords: BMI; biological profile; vitamin B12; Fe; and cortisol

\section{Introduction}

Malnutrition is widely regarded as unhealthy and a risk factor for being overweight or obese or underweight and developing several chronic diseases, including cancer, diabetes, and cardiovascular disease [1-3]. Obesity result from chronic consumption of a high-calorie, high-fat diet is a global disease, signifying one of the most significant warnings to global health $[1,2]$. Nutritional assessment is the study of anthropometric, biochemical, clinical, and dietary data to determine whether people or groups of people are well-nourished or malnourished and determine negative consequences [3-4]. An observational nutritional study is an epidemiological investigation study that does not include some intervention or experiment. Subjects are considered under natural living situations. Investigators may have to anticipate years for the results, except they use extensive continuous research, such as the National Health and Nutrition Examinations Survey (NHANES) [4-5]. Many volunteers and patients enter every year by responding to questions and sharing physical examinations. Scientists sift through data collected from NHANES to study all sorts of relationships between foods, dietary supplements, and health issues. For instance, NHANES data was used to conclude that folate and B-complex vitamin deficiency can lead to congenital disabilities, anemia, leukemia, stress, and depression in adults [5-7]. A nutritional assessment can be done through anthropometry, biochemical methods, clinical methods, and dietary method [7-9]. The present study aims to measure biochemical profile and level of Vitamin $\mathrm{B} 12, \mathrm{Fe}$, cortisol in the volunteer's population, and BMI in adult obese females and males subjects compared to the reference range.

\section{Subjects and methods}

\subsection{Subjects}

A total of ( 86 females and 65 males) volunteer were included in this study. Inclusion criteria were age 30- 52 years. Written and informed consent was taken from subjects. The studies were done according to the Helsinki Declaration. The appropriate institutional ethics committee approved the protocol and that it complied with the Helsinki Declaration as revised in 2013.

\subsection{Methods}

\subsubsection{Anthropometrics}


The subjects' weights and heights were evaluated on a single calibrated scale (SR Scales, SR Instruments). Anthropometric parameters were recorded while the subject was standing erect and barefoot. Height and weight were measured using standardized conventional methods. The formula calculated body mass index (BMI): weight in kilograms $(\mathrm{kg})$ divided by height in square meters $(\mathrm{m} 2)[1,2]$.

\subsubsection{Laboratory investigations}

\subsubsection{Biochemical analysis:}

Volunteer's blood sample was drawn as fasting blood samples at 8 am. The Access Vitamin B12 assay is a competitive binding immunoenzymatic assay at General Hospital Abu Arish. Serum aspartate aminotransferase (A.S.T.), alanine aminotransferase (A.L.T.), total bilirubin, albumin, fasting blood sugar (F.B.S.), creatinine, uric acid, total cholesterol, triglyceride, HDL, LDL were measured using standard Roche/Hitachi Cobas c 501 analyzers (Roche Diagnostics, Mannheim, Germany. Serum iron was tested using Dimension RXL Max (Dade Behring, USA) and automated analysers [1, 2, 8, and 9]. Cortisol level measured using MAGLUMI 600 instrumental in Abu-Arish general hospital Jazan in Saudi Arabia [8].

\subsubsection{Statistic}

Data were entered into the program in computer and analyzed using IBM SPSS software package version 20.0. (Armonk, NY: IBM Corp). We used The Kolmogorov-Smirnov, Shapiro, and D'agstino to check the normality of the distribution of variables, Studies between groups for categorical variables were evaluated utilizing the Chi-square test. ANOVA was applied to compare more than two groups for normally distributed quantitative variables and supported by the Post Hoc test for pairwise correlation. Kruskal Wallis test was applied to associate different groups for not normally distributed quantitative variables and served by the Post Hoc test (Dunn's for multiple comparisons test) for pairwise comparison. Pearson coefficient was applied to associate between quantitative variables. The significance of the obtained outcomes was resolved at the $5 \%$ level.

\section{Result}

Table 1 represented the data and results of the correlation between BMI and different parameters. According to Evans (1996) who suggests for the absolute value of r: 0.00-0.19: "very weak", 0.20-0.39: "weak", 0.40-0.59: "moderate", 0.60-0.79: "strong", 0.80-1.0: very strong. There is appositively relation between cortisol, liver and kidney profile, FBS, Cholesterol, triglyceride, LDL, and BMI. Other hand, there is a strong negative correlation between BMI, and Vitamin B12, Fe, and HDL.

Tables (2) show Comparison between female and male nutritional and biological profile. The data represented as Mean $\pm \mathrm{SD}$. BMI showed a significant difference in females $(32.6 \pm 4.5 \mathrm{~kg} / \mathrm{m} 2)$, and males $(28.5 \pm 2.7 \mathrm{~kg} / \mathrm{m} 2)$ at $\mathrm{p} \leq 0.05$, while WHO (World Health Organization's) recommended BMI for adult normal range 18.5-25 $\mathrm{kg} / \mathrm{m} 2$, Overweight $25-30 \mathrm{~kg} / \mathrm{m} 2$, Obese Class I 30-35 kg/m2, Obese Class II $35-40 \mathrm{~kg} / \mathrm{m} 2$, Obese Class III $>40 \mathrm{~kg} / \mathrm{m} 2$ sequentially. There is a significant rise of cortisol, lipid profile, FBS, liver, and kidney profile with BMI in the resulting table 2 work in females more than males compared to a reference range, and a significant decrease in Fe and vitamin B in the present study.

r: Pearson coefficient

*: Statistically significant at $\mathrm{p} \leq 0.05$

Evans (1996) suggests for the absolute value of $r$ :

0.00-0.19: "very weak", 0.20-0.39: “weak", 0.40-0.59: "moderate", 0.60-0.79: "strong", 0.80-1.0: “very strong"

Table 1. Correlation between BMI and different parameters $(n=151)$

\begin{tabular}{|l|c|c|c|}
\hline \multirow{2}{*}{ Normal range } & \multicolumn{2}{|c|}{ Studied population } \\
\cline { 3 - 4 } & & \multirow{2}{*}{ Female(n=86) } & \multirow{2}{*}{ Male (n=65) } \\
\cline { 3 - 4 } & & $32.6 \pm 4.5^{*}$ & $28.5 \pm 2.7^{*}$ \\
\hline BMI (kg/m $\mathbf{2})$ & $(185-624)$ & $427.2 \pm 2.81^{*}$ & $287.3 \pm 5.8^{*}$ \\
\hline Cortisol $(\mathbf{n m o l} / \mathbf{L})$ & $(\mathbf{1 3 3 - 6 7 5 )}$ & $253 \pm 52.2^{*}$ & $297 \pm 62.5^{*}$ \\
\hline Vitamin B12(pmol/L) & $(15-150)$ & $7.4 \pm 1.5^{*}$ & $9.9 \pm 1.5^{*}$ \\
\hline FST $(\boldsymbol{\mu g} / \mathbf{L})$ & $($ Up To 31) & $22.9 \pm 1.5^{*}$ & $21.5 \pm 1.6^{*}$ \\
\hline ALT $(\mathbf{U} / \mathbf{l})$ & (Up To 31) & $13.9 \pm 1.2^{*}$ & $21.0 \pm 4.2^{*}$ \\
\hline
\end{tabular}




\begin{tabular}{|l|c|c|c|}
\hline Albumin (g/dl) & $(3.5-5.4)$ & $4.1 \pm 1.2^{*}$ & $4.5 \pm 5.2^{*}$ \\
\hline FBS (mg/dl) & $(70-110)$ & $136.0 \pm 3.4^{*}$ & $120.1 \pm 2.7^{*}$ \\
\hline Creatinine (mg/dl) & $(0.5-0.9)$ & $0.7 \pm 1.7^{*}$ & $0.9 \pm 2.1^{*}$ \\
\hline Uric acid (mg/dl) & $(2-6)$ & $4.08 \pm 1.8^{*}$ & $4.69 \pm 2.1^{*}$ \\
\hline T-Cholesterol (mg/dl) & (Up to 200) & $219.3 \pm 2.6^{*}$ & $197.9 \pm 7.2^{*}$ \\
\hline Triglyceride (mg/dl) & (Up to 200) & $191.5 \pm 4.8^{*}$ & $190.5 \pm 5.2^{*}$ \\
\hline HDL (mg/dl) & (More than 35) & $41.1 \pm 2.7^{*}$ & $51.1 \pm 6.3^{*}$ \\
\hline LDL (mg/dl) & (Up to 140) & $141.7 \pm 3.2^{*}$ & $129.3 \pm 7.2^{*}$ \\
\hline
\end{tabular}

Data represented as Mean $\pm \mathrm{SD}, *$ is statistically significant at $\mathrm{p} \leq 0.05$.

Table 2. Comparison between before and female and male nutritional and biological profile

\section{Discussion}

Obesity is a complex condition involving an unnecessary amount of body fat. Obesity is not just a cosmetic matter; it is a medical obstacle that raises various diseases such as heart disorder, diabetes, high blood pressure, and specific cancers $[1,2]$. Although morbid obesity is associated with an excess of energy and macronutrient intake, it does not govern micronutrient deficiencies. However, even though obesity describes nutrition for caloric reasons, people with obesity can still be malnourished when it comes to micronutrients. Recent findings reported that that $13 \%$ had an iron deficiency in obese women, and $10 \%$ of them had a Vitamin B12 deficiency. In another study, $9.5 \%$ of obese individuals were deficient in Vitamin B12, 25\% in folic acid, 68\% in copper, and 74\% in zinc [10-11].

Moreover, recent investigators reported that $35 \%$ of obesity considering bariatric surgery patients were deficient in magnesium, 19\% in iron, and $17 \%$ in Vitamin A [12-13]. The previous outcomes result agrees with the present study. There is appositively relation between cortisol, liver and kidney profile, FBS, Cholesterol, triglyceride, LDL, and BMI. Other hand, there is a strong negative correlation between BMI, and Vitamin B12, Fe, and HDL. There is a significant elevation of cortisol, lipid profile, FBS, liver, and kidney function with BMI in the present work between females and males compared to a reference range and a significant decrease of $\mathrm{Fe}$ and vitamin $\mathrm{B}$.

Vitamin B12 has a vital role in the brain and nervous systems normal functioning and RBC and blood formation. Obesity and chronic stress lead to a decrease in Vitamin B12and Fe in the body by destructing the parietal cells (which secretes intrinsic factor for Vitamin B12absorption) in the stomach [14-15]. So, malabsorption of Vitamin B12occurs in the absence of intrinsic factors, leading to Vitamin B12 deficiency [16-17] that agrees with the present positive a negative correlation between BMI, vitamin12, and Fe outcomes. Further report finds that circulating cortisol and psychosocial stress may contribute to obesity and metabolic syndrome's pathogenesis [16] that connects with the present result, where there is a positive relationship between BMI and cortisol and lipid profile. Hamouda et al., 2016, 2018 find an appositive relation between cortisol, liver and kidney profile, FBS, Cholesterol, triglyceride, LDL, and obesity $[1,2]$. Serum vitamin B12 concentrations were lower among female obese adults than male-obese adults in the present study due to the inclusion criteria of BMI higher than females than males, which confirmed with different studied parameters. Besides, the effect of the menstrual cycle and hormone, which can be read by diet habits, and estrogen effects or genetic variations, are therefore hypothesized to play a role. The present result disagrees with previously reported that men are more susceptible to vitamin B12 deficiency among the healthy population [18].

Anemia is a health obstacle that occurs in developed countries. About 1.62 billion people undergo anemia worldwide [19]. Pregnant women are most susceptible to anemia during and after pregnancy, which can adversely affect the mother's health and fetus. Several research types have shown that $30.3 \%$ of maternal death situations due to bleeding at the time of childbirth and anemia during the pregnancy period are critical precipitating factors that indirectly become the cause of maternal death [20]. Anemia during pregnancy also makes premature birth, low birth weight babies, fetal complications, and infant mortality [21]. Anemia is when the decrease of red blood cells (erythrocytes) in the blood circulation or hemoglobin mass cannot perform its function as an oxygen carrier [22]. According to WHO (2011), iron is a common cause of anemia and iron; other nutritional deficiencies such as vitamin B12, vitamin $\mathrm{C}$, folic acid, vitamin $\mathrm{A}$, and malabsorption during obesity can also induce anemia. Iron is part of the hemoglobin molecule; with reduced iron, hemoglobin synthesis will decline, and the hemoglobin level will reduce. Hemoglobin is an element essential for the human body because lowering hemoglobin levels influence the ability to deliver oxygen $(\mathrm{O} 2)$ required by all body tissues. Iron with vitamin B12 is also one of erythrocyte formation [9, 21, and 22]. As in the present results, there is a negative relationship between BMI and Iron and vitamin B 12 that it was females' effect than males, which may be due to diet, malabsorption due to the accumulation of body fat, a different change in hormones and genetic of different sex [1, 2, 8, 9].

\section{Conclusion}

There is an appositively connection between BMI with cortisol, liver and kidney profile, FBS, Cholesterol, triglyceride, and LDL. Other support, there is a robust negative relationship between BMI and Vitamin B12, Fe, and HDL resulting from malabsorption in obesity condition. There is a significant elevation of cortisol, lipid profile, FBS, liver, and kidney function with BMI in the present work in females more than males compared to a reference range a and significant decrease in Fe and vitamin $\mathrm{B}$ that can be due to diet habits, BMI, hormones affect or genetic variations between male and female.

\section{Acknowledgements}

The authors thank Ibrahim Abdu Khardali, Ibraheem Mohammed Attafi, Mohsen M. Fageeh, Mohammad Ahmad Attafi, Magbool Essa Oraiby. They also want to bless all patients who trusted authors and were honest with them, making the research more accurate and useful. It will be a great pleasure to appreciate King Fahd hospital and General Hospital Abu Arish, Bish hospital and Poison Control and Medical Forensic Chemistry Center, Ministry of Health, Jazan in Saudi Arabia.

\section{Conflict of interest}

There is no conflict of interest to declare.

\section{References}

1. Hamouda AF, Abou El Noeman SA (2016) Effects of 6-Month Weight Loss New Program on Anthropometric Measurements and Biological Profile. Journal of Pharmacy and Pharmacology 4, 2338.

2. Hamouda AF, Abou El Noeman SA, Khardalic IA, et al(2018) Study the Association Between Diet Program on Human Semen, Biological Profile, and Anthropometric Measurements in Obese 
Men. International Journal of Nutrition and Food Sciences; 7(1): 24-29.

3. Crawford F., Mackison D., Mooney JD. et al. (2017) Observation and assessment of the nutritional quality of 'out of school' foods popular with secondary school pupils at lunchtime. BMC Public Health 17, 887.

4. Carmel R. (2008) Efficacy and safety of fortification and supplementation with vitamin B12: Biochemical and physiological effects. Food Nutr Bull; 29(2Suppl):S177-87.

5. Bhatia P, Kulkarni JD, Pai SA. (2012) Vitamin B12 deficiency in India: Mean corpuscular volume is an unreliable screening parameter. Natl Med J India; 25: 336-8.

6. Premkumar M, Gupta N, Singh T, Velpandian T. (2012) Cobalamin and folic acid status in relation to the etiopathogenesis of pancytopenia in adults in a tertiary care centre in North India. Anemia 2012:707402.

7. Khanduri U, Sharma A. (2007) Megaloblastic anemia: Prevalence and causative factors. Natl Med J India 20:172-5.

8. Hamouda AF, Ashraf MH, Ehab YE, et al. (2020) Short Communication Pilot Study on Stress and Its Chronic Consequences of College Students. CPQ Nutrition 4:2.1-12.

9. Hamouda AF, Moustafa Hassan AA, Khardali IA, et al. (2019) A Screening Pilot Study on the Relation Between Body Mass Index, Heavy Metal and Mineral Levels in College Students. Electronic J Biol. 15:3.

10. Sánchez A, Rojas P, Basfi-Fer K, Carrasco F, Inostroza J, Codoceo J, Valencia A, Papapietro K, Csendes A, Ruz M.(2015) Micronutrient Deficiencies in Morbidly Obese Women Prior to Bariatric Surgery. Obes Surg Feb; 26(2):361-8.

11. de Luis DA, Pacheco D, Izaola O, Terroba MC, Cuellar L, Cabezas G. (2013) Micronutrient status in morbidly obese women before bariatric surgery. Surg Obes Relat Dis 9(2):323-7.

12. Lefebvre P, Letois F, Sultan A, Nocca D, Mura T, Galtier F.(2011)Nutrient deficiencies in patients with obesity considering bariatric surgery: a cross-sectional study. Surg Obes Relat Dis. 10(3):540-6.
13. Allen RH, Stabler SP, Savage DG, Lindenbaum J. (1993) Metabolic abnormalities in cobalamin (vitamin B12) and folate deficiency. FASEB J 7: 1344-1353.

14. Hughes CF, Ward M, Hoey L, McNulty H. (2013) Vitamin B12 and ageing: current issues and interaction with folate. Ann Clin Biochem; 50: 315-329.

15. Lodhi R, Panchal A. (2014) Interrelationship of Vitamin B12, Androgens and Cortisol in Chronic Stress and associated Vascular Dysfunction. J Pharmacol Clin Toxicol 2(2):1028.

16. Abraham SB, Rubino D, Sinaii N, Ramsey S, and Nieman LK.(2012) Cortisol, obesity and the metabolic syndrome: A crosssectional study of obese subjects and review of the literature Obesity (Silver Spring); 21(1): E105-E117.

17. Yangbo S, Minxian S, ,Buyun L, Yang D, et al.(2019) Inverse Association Between Serum Vitamin B12 Concentration and Obesity Among Adults in the United States. Front Endocrinol (Lausanne) 10: 414. Published online 2019 Jun 27.

18. Margalit I, Cohen E, Goldberg EK. (2018) Vitamin B12 Deficiency and the Role of Gender: A Cross-Sectional Study of a Large Cohort. Ann Nutr Metab 72:265-271.

19. WHO. (2011). Worldwide Prevalence of Anemia: WHO Global Database on Anemia. Geneva, witzerland: World Health Organization Press

20. Citra DA, Arni AD. (2018) Relationship of Ferritin Levels and Vitamin B12 Levels Pregnant Women Anemia on Baby Birth Weight. Journal of Midwifery Vol 3: No 2.

21. Gebre. A dan Mulugeta. A. (2015) Prevalence of Anemia and Associated Factors among Pregnant. Women in North Western Zone of Tigray, Northern Ethiopia: A Cross-Sectional Study, Journal Of Nutrition and Metabolism, 2015, pp. 1-7

22. Visnjevac N, Segedi LM, Curcic A, Visnjevac J, Stajic D.(2011) Blood ferritin levels in pregnant women and prediction of the development of fetal intrauterine growth restriction, $J$ Med Biochem, Vol. 30, No. 4, pp. 317-322. 